\title{
Neural Basis of Aging: The Penetration of Cognition into Action Control
}

\author{
Sofie Heuninckx, ${ }^{1}$ Nicole Wenderoth, ${ }^{1}$ Filiep Debaere, ${ }^{1}$ Ronald Peeters, ${ }^{2}$ and Stephan P. Swinnen ${ }^{1}$ \\ ${ }^{1}$ Motor Control Laboratory, Department of Kinesiology, Katholieke Universiteit Leuven, B-3001 Heverlee, Belgium, and ${ }^{2}$ Department of Radiology, \\ Universitair Ziekenhuis Gasthuisberg, B-3000 Leuven, Belgium
}

\begin{abstract}
Although functional imaging studies have frequently examined age-related changes in neural recruitment during cognitive tasks, much less is known about such changes during motor performance. In the present study, we used functional magnetic resonance imaging to investigate age-related changes in cyclical hand and/or foot movements across different degrees of complexity. Right-handed volunteers (11 young, 10 old) were scanned while performing isolated flexion-extension movements of the right wrist and foot as well as their coordination, according to the "easy" isodirectional and "difficult" nonisodirectional mode. Findings revealed activation of a typical motor network in both age groups, but several additional brain areas were involved in the elderly. Regardless of the performed motor task, the elderly exhibited additional activation in areas involved in sensory processing and integration, such as contralateral anterior insula, frontal operculum, superior temporal gyrus, supramarginal gyrus, secondary somatosensory area, and ipsilateral precuneus. Age-related activation differences during coordination of both segments were additionally observed in areas reflecting increased cognitive monitoring of motor performance, such as the pre-supplementary motor area, pre-dorsal premotor area, rostral cingulate, and prefrontal cortex. In the most complex coordination task, the elderly exhibited additional activation in anterior rostral cingulate and dorsolateral prefrontal cortex, known to be involved in suppression of prepotent response tendencies and inhibitory cognitive control. Overall, these findings are indicative of an age-related shift along the continuum from automatic to more controlled processing of movement. This increased cognitive monitoring of movement refers to enhanced attentional deployment, more pronounced processing of sensory information, and intersensory integration.
\end{abstract}

Key words: aging; fMRI; motor control; interlimb coordination; cognition; cyclical movement

\section{Introduction}

It is well documented that normal aging is associated with deterioration in cognitive (Park et al., 2003; Hedden and Gabrieli, 2004) and motor functioning (Spirduso, 1982; Welford, 1988; Seidler and Stelmach, 1995). Particularly with respect to cognitive aging, neuroimaging studies have demonstrated repeatedly that older individuals demonstrate differential activity in the same brain areas or use other brain areas when compared with younger subjects, even when behavioral performance is matched (Grady, 2000; Cabeza, 2001; Reuter-Lorenz, 2002; Hedden and Gabrieli, 2004). These altered brain activity patterns in the elderly possibly represent adaptive plasticity within the face of agerelated neurodegenerative and neurochemical changes in the brain to maintain performance (Grady et al., 1994; Cabeza et al., 1997; Buckner, 2004).

Although cognitive aspects have been studied quite fre-

Received April 1, 2005; revised June 7, 2005; accepted June 7, 2005.

This work was supported by a grant from the Research Council of Katholieke Universiteit Leuven, Belgium (Contract 0T/03/61), and by the Flanders Fund for Scientific Research (Projects G.0460.04 and G.0105.05). S.H. was funded by a fellowship from Fonds voor Wetenschappelijk Onderzoek Vlaanderen.

Correspondence should be addressed to Stephan P. Swinnen, Laboratory of Motor Control, Group Biomedical Sciences, Katholieke Universiteit Leuven, Tervuursevest 101, B-3001 Heverlee, Belgium. E-mail: Stephan.Swinnen@faber.kuleuven.be.

DOI:10.1523/JNEUROSCI.1263-05.2005

Copyright $\odot 2005$ Society for Neuroscience $\quad$ 0270-6474/05/256787-10\$15.00/0 quently, much less is known about age-related changes in brain activity during motor tasks. First, neuroimaging studies revealed that elderly subjects exhibit altered brain activation patterns during simple isolated finger movements (Calautti et al., 2001; Hutchinson et al., 2002; Mattay et al., 2002) or hand movements (Hutchinson et al., 2002; Ward and Frackowiak, 2003). However, it remains essentially unresolved which brain areas are recruited by the elderly during the performance of more complex cyclical interlimb coordination tasks. Coordination is of primary concern to aging adults, because it is intrinsic to many activities of daily living, such as walking, dressing, and driving a vehicle. The loss of the ability to produce smooth coordinated muscle activity will result in a higher risk of falling (de Rekeneire et al., 2003) and a decreased functional and social independence (Spirduso, 1995). Moreover, previous work has shown that different control mechanisms emerge during the coordination of two or more limbs compared with isolated limb movements. Thus, although elderly subjects and pathological groups often produce isolated limb movements successfully, they encounter pronounced difficulties when limbs have to be coordinated (Swinnen, 2002).

In the present study, we used functional magnetic resonance imaging (fMRI) to identify, for the first time in seniors, the neural basis of complex interlimb movement patterns, which require similar coordinative mechanisms as locomotion. Brain activations in young and older adults were compared during the per- 
formance of isolated rhythmical hand/foot movements as well as during their coordination, whereby both limbs moved either in the same direction (isodirectional) or in opposite directions (nonisodirectional). Previous behavioral work has shown convincingly that nonisodirectional patterns are more difficult and are produced with lower accuracy and stability than isodirectional patterns in normal subjects (Baldissera et al., 1982, 1991; Kelso and Jeka, 1992; Carson et al., 1995; Serrien et al., 2000) and in Parkinson and stroke patients (Swinnen et al., 1997; Debaere et al., 2001b). Accordingly, the aforementioned conditions represented a gradient of complexity, ranging from isolated to coordinated limb movements of different difficulty levels.

We hypothesized that isolated, as well as coordinated, hand and foot movements would activate a network, which is more widespread and reflects increased recruitment of cognitive resources in the elderly compared with the young subjects. Furthermore, age-related activation differences were expected to increase as a function of task difficulty, primarily in nonmotor areas.

\section{Materials and Methods}

Participants

Twenty-four volunteers participated in the study, including 12 young adults (mean, 22.4 years of age; range, 20-25 years of age; six women and six men) and 12 older adults (mean, 64.8 years of age; range, 62-71 years of age; six women and six men). Imaging data of one young and two older subjects contained artifacts and were excluded from all analyses. All participants were right-handed, as assessed by the Edinburgh Handedness Inventory (Oldfield, 1971). None reported a history of neurological disease or skeletomotor dysfunction or was taking psychoactive or vasoactive medication. General cognitive functions were assessed using the Mini-Mental State Examination (Folstein et al., 1975). All participants scored within normal limits (score $\geq 26$ ). Participants were informed about the experimental procedures and provided written informed consent. The study design was approved by the local Ethics Committee of Biomedical Research at Katholieke Universiteit Leuven and was performed in accordance with the ethical standards of the 1964 Declaration of Helsinki.

\section{Experimental design}

Task. The participants performed five different conditions consisting of four movement tasks and one rest (REST) condition in which no movements were performed. The movement tasks were composed of isolated cyclical flexion-extension movements of the right wrist (HAND) and right ankle (FOOT) and cyclical coordination of the hand and foot according to the isodirectional (ISODIR) and nonisodirectional (NONISODIR) mode. During ISODIR coordination, both limb segments were moved in the same direction (i.e., hand flexion together with foot flexion). During NONISODIR coordination, segments were moved in opposite directions (i.e., hand flexion combined with foot extension) (Fig. 1). This set of conditions enabled us to assess age-related brain activations across different degrees of coordinative complexity. To our knowledge, this is the most complex gross motor task that has been studied so far in elderly subjects using medical imaging technologies. It also shares some similarities with locomotion.

During scanning, participants lay supine inside the scanner. The lower legs were supported by a cushion to ensure free-ankle rotation. The right arm was extended along the trunk, and the distal part of the arm was supported to enable free movements of the wrist. The wrist and foot were positioned in a nonferromagnetic wrist-hand and ankle-foot orthosis, respectively. Movements were restricted to the sagittal plane. The frictionless axis of the orthosis was aligned with the anatomical axis of the

joint such that movements were not hindered. Angular displacements of the joints were registered by means of high-precision shaft encoders (HP; 4096 pulses per revolution; sampled at $100 \mathrm{~Hz}$ ) fixed to the movement axis of the orthosis. This nonferromagnetic kinematic registration device enabled us to register movements on-line during brain scanning and free of interference. It allowed a direct mapping between obtained kinematics and brain scanning. Movements were limited to the wrist and ankle, whereas the other segments were kept still. A bite-bar was used to minimize head motion. Subjects were trained to look at a fixation cross, which was displayed in front of them at all times.

All five conditions were paced by an electronic metronome (DTM-12; KORG, Tokyo, Japan), whereby a full movement cycle was completed on every second beat (one beat for peak flexion and one for peak extension). The older adults performed five scanning runs at a movement frequency of $1 \mathrm{~Hz}$, whereas the younger adults performed 10 runs, five at a movement frequency of $1 \mathrm{~Hz}$ and five at a movement frequency of $1.5 \mathrm{~Hz}$. For purposes of equating difficulty level, however, we compared the older subjects' performance at $1 \mathrm{~Hz}$ to that of the younger subjects at $1.5 \mathrm{~Hz}$. Indeed, previous work demonstrated that these cycling frequencies represented a comparable ratio to the maximal frequency at which these patterns could be performed successfully by both groups (Heuninckx et al., 2004). Between the different scanning runs, rest periods of 3 min were provided.

One or $2 \mathrm{~d}$ before the experimental session, a $45 \mathrm{~min}$ practice was provided in a dummy-scanner to ensure correct performances. For all four movement conditions, participants were trained to move their hand and foot, respectively, with amplitudes between 20 and $30^{\circ}$. Additionally, subjects were trained to avoid eye movements and to look at the fixation cross.

Scanning procedure. The MR images were acquired in a 3-T Intera MR scanner (Philips, Best, The Netherlands), using a six-element SENSE head coil (MRI Devices, Waukesha, WI). Functional time series consisted of 105 whole-brain gradient-echo echoplanar images (EPIs) [repetition time (TR)/echo time (TE), 3000/33 ms; field of view, $230 \mathrm{~mm}$; matrix, $112 \times 112$; slice thickness, $4.0 \mathrm{~mm}$; interslice gap, $0.4 \mathrm{~mm}$; 34 sagittal slices; SENSE factor, 2]. Each time series contained three blocks of the five conditions. Each condition lasted for $21 \mathrm{~s}$ (corresponding to seven whole-brain images) and was triggered by a visual template displaying the task to be performed. The different task conditions were randomized across subjects and runs. Each scanning session ended with the acquisition of a three-dimension SENSE high-resolution T1weighted image (TR/TE, 9.68/4.6 ms; inversion time, $1100 \mathrm{~ms}$; field of view, $250 \mathrm{~mm}$; matrix, $256 \times 256$; slice thickness, $1.2 \mathrm{~mm}$; 182 slices; SENSE factor, 2) for anatomical detail.

\section{Data analyses}

Kinematic analysis. The coordination between the limb segments was assessed by means of a relative phase measure, which is the subtraction of the phase angles of each limb according to the following formula: $\Phi=\theta_{\mathrm{w}}$ $-\theta_{\mathrm{f}}=\tan ^{-1}\left[\left(d X_{\mathrm{w}} / d t\right) / X_{\mathrm{w}}\right]-\tan ^{-1}\left[\left(d X_{\mathrm{f}} / d t\right) / X_{\mathrm{f}}\right]$, where $\mathrm{w}$ and $\mathrm{f}$ denote wrist and foot, respectively, $\theta_{\mathrm{w}}$ refers to the phase of the wrist movement at each sample, $X_{\mathrm{w}}$ is the position of the wrist after rescaling to the interval $[-1,1]$ for each cycle of oscillation, and $d X_{\mathrm{w}} / d t$ is the normalized 
Table 1. Kinematic data

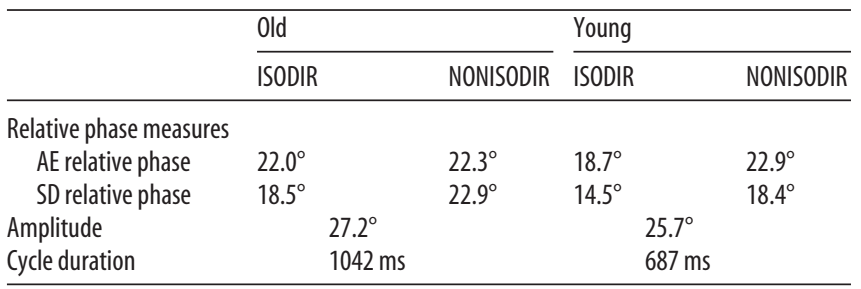

AE, Absolute error. tests were performed when necessary for significant interactions. The $\alpha$-level of significance was set to $\alpha=0.05$.

Imaging analysis. Imaging data were analyzed with Statistical Parametric Mapping 2 (SPM2) (Wellcome Department of Cognitive Neurology, London, UK) implemented in MatLab 6.5 (MathWorks, Natick, MA). For each subject, all EPI volumes were realigned to the first volume of the first time series, and a mean image of the realigned volumes was created. This mean image was smoothed with a Gaussian kernel of $6 \mathrm{~mm}$ full width at half maximum (FWHM) and coregistered to the anatomical T1-weighted image. To normalize the anatomical image as well as the EPIs to a standard reference system

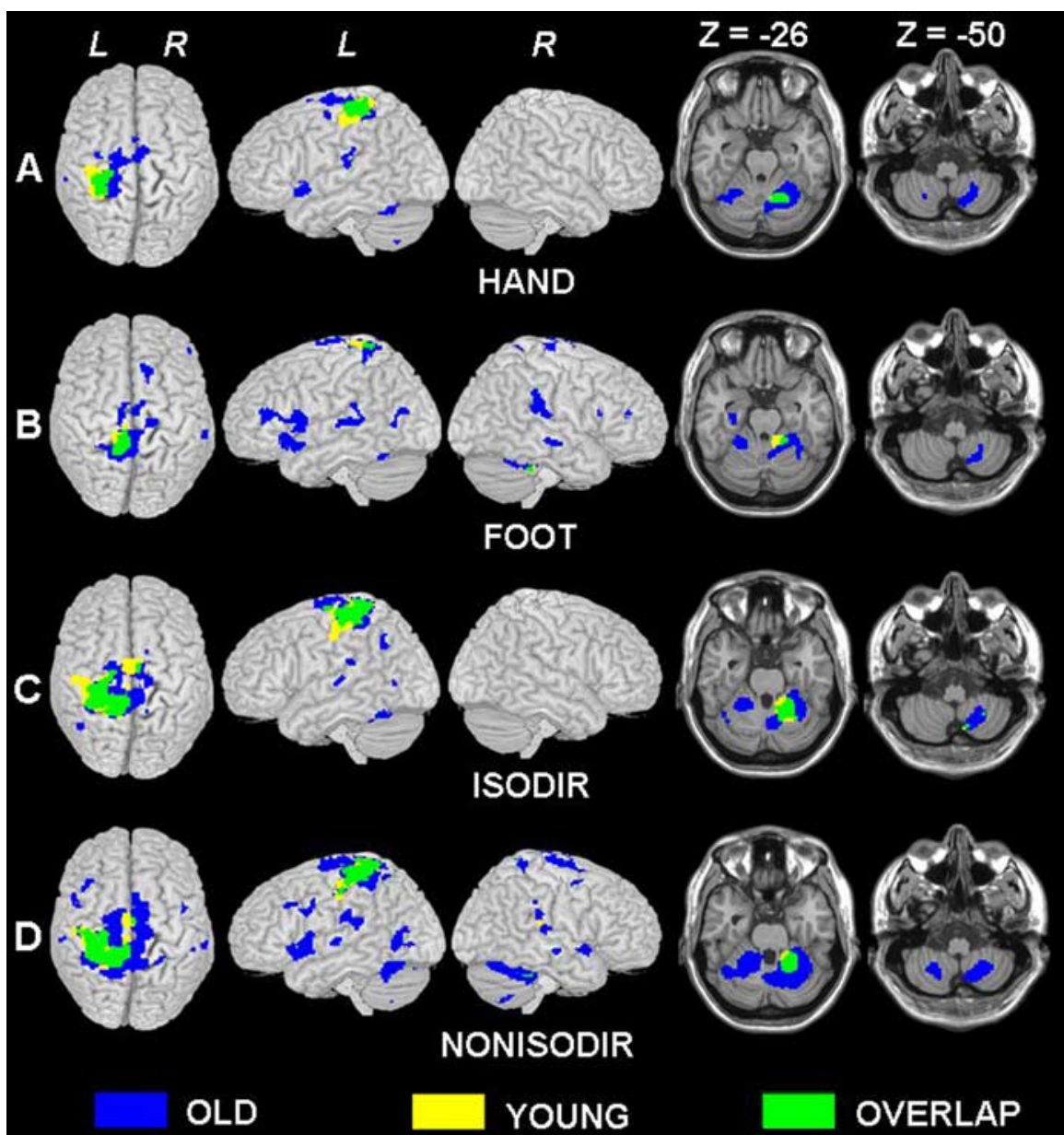

Figure 2. Group mean activations for each motor task ( $\boldsymbol{A}$, HAND; $\boldsymbol{B}$, FOOT; $\boldsymbol{C}$, ISODIR coordination; $\boldsymbol{D}$, NONISODIR coordination) versus REST overlaid on standard MNI renders and templates. Significant voxels ( $p<0.001$; corrected for multiple comparisons) are indicated in blue for the old group, in yellow for the young group, and in green for overlap between activation of both groups; in each, the height threshold is $t=6.98$. L, Left hemisphere; $R$, right hemisphere.

(Talairach and Tournoux, 1988), the following procedure was applied. First, the anatomical image as well as a representative template image [Montreal Neurological Institute (MNI)] were segmented into gray matter, white matter, and CSF. Then, the anatomical gray matter image was smoothed $(6 \mathrm{~mm}$ FWHM) and normalized to the gray matter of the MNI brain. Subsequently, the derived normalization parameters were applied to the EPI images, which were subsampled to a voxel size of $2 \times 2 \times 2 \mathrm{~mm}$ and smoothed with a Gaussian kernel of 10 mm FWHM.

All statistical analyses were performed in the context of the general linear model (Friston et al., 1995a,b). Each condition was modeled using a delayed boxcar function convolved with the SPM2 hemodynamic response function. An appropriate high-pass filter was applied to remove low-frequency drifts. Additionally, movement parameters derived from realignment were added as covariates of no interest to correct for confounding effects induced by head movement. Contrasts of interest were first estimated for each subject individually (averaging activation across runs) and then subjected to a second-level, random-effects analysis.

In the first step, within-group activation was calculated by contrasting each movement condition with REST and applying a second-level $t$ test. The results of these analyses were corrected for multiple comparisons by applying a false discovery rate (FDR) procedure (Genovese et al., 2002). Note that the FDR correction is adaptive to the signal extent, which can result in different $t$ thresholds for the calculated contrasts. To overcome this problem, we calculated a common $t$ threshold ( $t>6.98$ ) across all contrasts of interest, ensuring an overall FDR with $p<0.001$ (http://www.sph.umich.edu/ nichols/FDR/multFDR.m). The resulting 0.001 FDR threshold controls the fraction of false positives among all of the suprathreshold voxels in all contrasts considered. The minimum cluster

instantaneous velocity. Absolute deviations from the target relative phase (i.e., 0 and $180^{\circ}$ for ISODIR and NONISODIR coordination, respectively) were calculated to obtain a measure of relative phase accuracy (phase error). The SD of relative phase was used as an estimate of movement pattern stability. In addition to the relative phase measures, cycle durations and amplitude of the limb movements were quantified. Cycle duration was defined as the time that elapsed between successive peak extension positions. The spatial measure consisted of the absolute value of the peak-to-peak amplitude for each individual cycle.

For the statistical analysis, the above parameters were determined for each condition and subsequently averaged across repetitions and runs. All statistical analyses consisted of repeated-measures ANOVAs with the between-factor group (young, old) and the within-factors mode (single limb, ISODIR, NONISODIR) and limb (hand, foot). Post hoc Tukey's size was set to 10 voxels.

In a second step, between-group comparisons were made by means of independent $t$ tests. To compare which areas were differentially activated in old versus young subjects for isolated limb movements, the following conjunction was calculated: [(Hand-Rest $)_{\text {old }}$ versus (Hand-Rest $\left.)_{\text {young }}\right]$ $\left.\cap\left[(\text { Foot-Rest })_{\text {old }} \text { versus (Foot-Rest }\right)_{\text {young }}\right]$. Similarly, to identify which areas were differentially activated in old versus young subjects during hand-foot coordination, the following conjunction was calculated: $\left.\left[(\text { ISODIR-Rest })_{\text {old }} \text { versus (ISODIR-Rest }\right)_{\text {young }}\right] \cap$ [(NONISODIRRest) old versus (NONISODIR-Rest) young $]$. Finally, we were interested in between-group differences arising from the comparison of the nonisodirectional with the isodirectional hand-foot movements. Therefore, we applied an independent $t$ test to the (NONISODIR-ISODIR) old versus (NONISODIR-ISODIR) young contrast. For all between-group compari- 
sons, FDR correction was applied, ensuring an overall $p<0.05$ (i.e., $t>3.60$ ).

\section{Results}

\section{Kinematic data}

The kinematic data were successfully recorded on-line during the fMRI scanning sessions. Mean values of movement accuracy, stability, amplitude, and cycle duration are shown for both groups in Table 1 .

\section{Relative phase measures}

Analyses of relative phase error (coordination accuracy) and analysis of the SD of relative phase (coordination stability) were assessed by a $2 \times 2$ (group $\times$ coordination mode) ANOVA with repeated measures on the last factor. The analysis of relative phase error did not reveal any significant effect, which indicates that there were no significant differences between both groups $\left(F_{(1,19)}=0.4 ; p=0.54\right)$ and between coordination modes $\left(F_{(1,19)}=2.4 ; p=0.13\right)$. In contrast, the coordination stability analysis revealed a significant main effect of coordination mode $\left(F_{(1,19)}=9.9 ; p<0.01\right)$ and group $\left(F_{(1,19)}=6.5 ; p<0.05\right)$. In accordance with the literature, ISODIR coordina-

tion was performed with higher stability than NONISODIR coordination. Coordination performance of the older participants was slightly less stable than that of the younger subjects, notwithstanding the fact that different cycling frequencies were used to correct for task difficulty levels between both groups (i.e., $1 \mathrm{~Hz}$ vs $1.5 \mathrm{~Hz}$ for the elderly vs young adults, respectively).

\section{Amplitude and cycle duration measures}

Analysis of amplitude was assessed by a $2 \times 2 \times 3$ (group $\times$ limb $\times$ mode) ANOVA with repeated measures on the last two factors. Although this analysis revealed small but significant amplitude variations across limbs $\left(F_{(1,19)}=18.3 ; p<0.0005\right.$; hand amplitude, $24.0^{\circ}$; foot amplitude, $\left.28.9^{\circ}\right)$ and modes $\left(F_{(2,38)}=\right.$ 11.6; $p<0.0005$; single limb, $26.4^{\circ}$; ISO, $25.1^{\circ}$; NONISO, $27.7^{\circ}$ ), no significant differences between groups were determined $\left(F_{(1,19)}=0.8 ; p>0.05\right)$.

Analysis of cycle duration revealed that both the younger and older subjects complied well with the rhythm of the metronome across all conditions.

\section{fMRI data}

Group mean versus rest contrasts for isolated limb movements Areas significantly activated by isolated HAND and FOOT movements compared with REST are shown in Figure 2, $A$ and $B$, for both the old (blue) and the young (yellow) subjects (for coordinates and $t$ values, see supplemental Tables $1^{\star}$ and $2^{\star}$, available at www.jneurosci.org as supplemental material).

In the young group, the activation pattern for HAND movement (Fig. 2A) consisted only of the left primary sensorimotor cortex (SM1) and right anterior cerebellum. The same brain areas were also activated in the elderly during HAND movement (Fig. $2 A$, green overlap). However, even for isolated HAND movement, the activation pattern in the elderly was more extended (i.e., SM1 activation extended to precentral midline areas) and additional brain regions were involved [e.g., left anterior insular cortex, dorsal premotor area (PMd), and secondary somatosen-
Table 2. Brain areas with significantly larger activation in the old group during isolated hand and foot movements

\begin{tabular}{|c|c|c|c|c|c|}
\hline Area activated & Side & $x$ & $y$ & $z$ & $t$ value \\
\hline Anterior insular cortex & $\mathrm{L}$ & & 24 & & 6.10 \\
\hline Inferior frontal gyrus pars & & -36 & & -8 & \\
\hline opercularis & $\mathrm{L}$ & -50 & 20 & 18 & 4.52 \\
\hline Superior temporal gyrus & L & -60 & & & 4.02 \\
\hline Inferior postcentral sulcus & & & -12 & -2 & \\
\hline$(\mathrm{S} 2)$ & $\mathrm{L}$ & -58 & -12 & 26 & 4.06 \\
\hline Supramarginal gyrus & $\mathrm{L}$ & -62 & -28 & 28 & 4.30 \\
\hline Fusiform gyrus & L & -46 & -44 & & 4.39 \\
\hline Precuneus (superior parietal & & & & -18 & \\
\hline gyrus) & $\mathrm{R} / \mathrm{M}$ & 8 & -48 & 68 & 4.46 \\
\hline
\end{tabular}

$t$ values and localizations (MNI coordinates) of activation peaks showing significantly $(p<0.05$; corrected for multiple comparisons) larger activation in the old group compared with the young group resulting from the following conjunction analysis: [(Hand-Rest) ${ }_{\text {old }}$ Vs (Hand-Rest $\left.\left.)_{\text {young }}\right] \cap\left[(\text { Foot-Rest })_{\text {old }} \text { Vs (Foot-Rest }\right)_{\text {young }}\right]$. L, Left hemisphere; $\mathrm{R}$, right hemisphere; $\mathrm{M}$, medial; $\$ 2$, secondary somatosensory area.

sory area (S2)]. For the cerebellum, the elderly but not the young subjects exhibited bilateral activation in the anterior and posterior lobe.

For isolated FOOT movements (Fig. 2B), a similar picture of results was observed. The activation pattern of the older group was even more diffuse than for the HAND movements, and some additional regions were activated bilaterally [e.g., inferior frontal gyrus pars opercularis (IFGPO), inferior frontal sulcus, S2, and anterior cerebellar regions].

These differences between the young and the old subjects were studied in additional detail by means of a statistical analysis directly contrasting the isolated limb movements between both groups.

Old-young group differences during isolated limb movements Group differences in task-related blood oxygenation level dependent (BOLD) responses during isolated limb movements were studied by means of a conjunction analysis, which identifies 


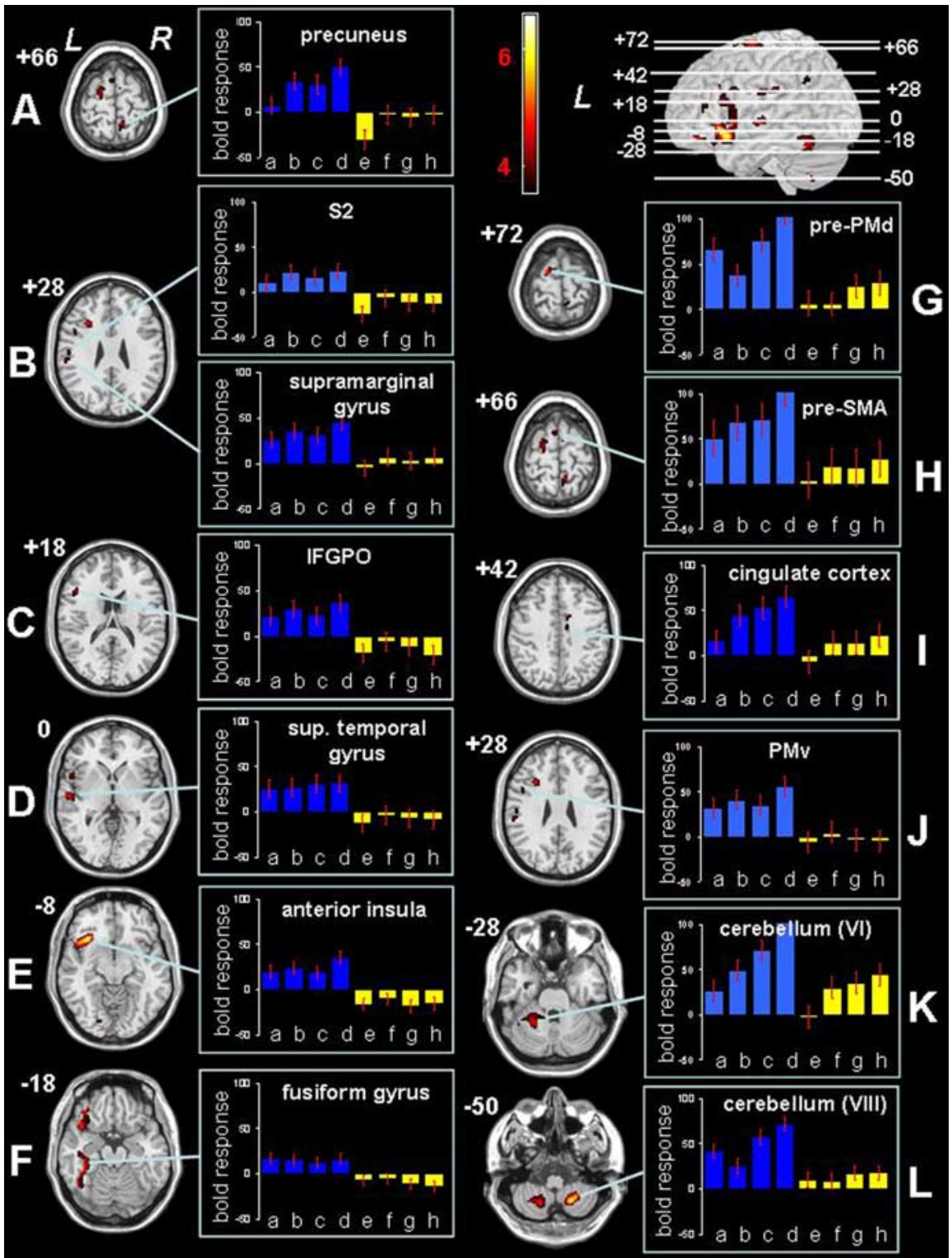

Figure 4. $\quad A-L$, Statistical parametric mappings representing significantly larger activation in the old group compared with the young group during ISODIR and NONISODIR coordination, resulting from the following conjunction analysis: [(ISODIR-Rest) ${ }_{\text {old }}$ vs (ISODIR-Rest $\left.)_{\text {young }}\right] \cap\left[\left(\right.\right.$ NONISODIR-Rest) ${ }_{\text {old }}$ vs (NONISODIR-Rest) ${ }_{\text {young }}$. Significant voxels $(p<0.05$; corrected for multiple comparisons) are indicated in the red spectrum, and the height threshold is $t=3.60$. L, Left hemisphere; $R$, right hemisphere; sup. temporal gyrus, superior temporal gyrus. Bar plots show the estimated BOLD responses in arbitrary units for the different movement conditions and for both groups: $a$, old hand; $b$, old foot; c, old ISODIR; $d$, old NONISODIR; e, young hand; $f$, young foot; $g$, young ISODIR; h, young NONISODIR.

between-group differences in those regions that are common to both isolated limb movement conditions.

Compared with the young group, the pattern of activation in the old group was more widespread (Fig. 3, Table 2). The old group exhibited significantly higher activation than the young group in a relatively large cluster with peak activation in the left anterior insular cortex (Fig. $3 E$ ). In addition, larger activation for the old group was observed in the left IFGPO (Fig. 3C), left superior temporal gyrus (Fig. 3D), left inferior postcentral sulcus/S2 (Fig. 3B), left supramarginal gyrus (Fig. 3B), and left fusiform gyrus (Fig. $3 F$ ). Finally, the old group exhibited significantly higher activation than the young group in a superior parietal cluster with peak activation in the right precuneus (Fig. 3A).

No region was significantly more activated in the younger group than in the older group.
Group mean versus rest contrasts for coordination patterns

The general network for producing the hand-foot coordination patterns was established first in both experimental groups before identifying between-group differences in brain activation (Fig. 2C,D) (supplemental Tables $3^{\star}$ and $4^{\star}$, available at www.jneurosci.org as supplemental material).

In the younger group, performing the ISODIR coordination pattern involved primarily the same brain areas as for isolated limb movements (i.e., left SM1 and right anterior cerebellum) albeit in larger clusters. Furthermore, some additional areas were activated [i.e., supplementary motor area (SMA), caudal cingulate cortex (CCC), left PMd, and right posterior cerebellum] (Fig. 2C). The same brain areas were also activated in the elderly during ISODIR coordination (Fig. 2C, green overlap). However, the activation pattern in the elderly was more extended, and additional regions were involved (e.g., superior temporal, angular, and fusiform gyrus).

Performance of NONISODIR coordination in the young group resulted in a similar pattern of results (Fig. 2D) as for ISODIR coordination. However, the activation pattern of NONISODIR coordination in the elderly (Fig. 2D) was much more extended, and additional regions were recruited, including the inferior frontal sulcus, middle frontal gyrus, rostral cingulate cortex (RCC), and supramarginal and middle occipital gyrus. Moreover, during NONISODIR but not during ISODIR, some of the additionally involved regions were activated bilaterally, including the superior temporal gyrus, superior parietal gyrus, S2, and anterior and posterior cerebellar regions.

\section{Old-young group differences for}

coordination movements

Group differences in brain activation during the performance of ISODIR and NONISODIR coordination patterns were studied by means of a conjunction analysis, which yields between-group differences common to both coordination modes.

Compared with the young group, the pattern of activation in the old group was more widespread (Fig. 4, Table 3). The old group exhibited significantly larger activation than the young group in the left anterior insular cortex (Fig. $4 E$ ), left IFGPO (Fig. $4 C$ ), left S2 (Fig. 4B), left superior temporal gyrus (Fig. 4D), left supramarginal gyrus (Fig. $4 \mathrm{~B}$ ), left fusiform gyrus (Fig. $4 F$ ), and right precuneus (Fig. 4A). Remarkably, these seven regions demonstrated already significantly higher activation in older subjects compared with younger subjects in the isolated limb conjunction analysis (Fig. 3, Table 2). The bar plots in Figure 4 display the estimated BOLD responses for the four different movement con- 


\begin{tabular}{|c|c|c|c|c|c|}
\hline Area activated & Side & $x$ & $y$ & $z$ & $t$ value \\
\hline \multicolumn{5}{|l|}{ Inferior frontal sulcus } & 6.56 \\
\hline & L & -30 & 20 & 26 & 4.79 \\
\hline & $\mathrm{L}$ & -44 & 36 & 14 & 3.96 \\
\hline \multicolumn{6}{|l|}{ Inferior frontal gyrus pars } \\
\hline $\begin{array}{l}\text { opercularis } \\
\text { Inferior precentral sulcus }\end{array}$ & L & -50 & 20 & 18 & 4.27 \\
\hline$(\mathrm{PMv})$ & L & -50 & 10 & 28 & 3.87 \\
\hline \multicolumn{6}{|l|}{ Superior frontal gyrus } \\
\hline \multirow[t]{2}{*}{ (pre-SMA) } & $L / M$ & -6 & 10 & 68 & 4.18 \\
\hline & $\mathrm{R} / \mathrm{M}$ & 4 & 0 & 66 & 3.75 \\
\hline $\begin{array}{l}\text { Rostral cingulate cortex } \\
\text { Superior frontal gyrus }\end{array}$ & $\mathrm{R}$ & 10 & 4 & 42 & 4.05 \\
\hline (PMd) & L & -20 & -6 & 72 & 5.16 \\
\hline Superior temporal gyrus & L & -58 & -14 & 0 & 4.77 \\
\hline \multicolumn{6}{|l|}{ Inferior postcentral sulcus } \\
\hline$(\mathrm{S} 2)$ & L & -58 & -14 & 26 & 4.03 \\
\hline Supramarginal gyrus & L & -60 & -28 & 28 & 4.16 \\
\hline Fusiform gyrus & L & -44 & -42 & -18 & 4.78 \\
\hline \multicolumn{6}{|l|}{ Precuneus (superior pari- } \\
\hline etal gyrus) & $\mathrm{R} / \mathrm{M}$ & 8 & -48 & 66 & 4.54 \\
\hline Angular gyrus & L & -44 & -56 & 38 & 3.71 \\
\hline Cerebellar vermis (IV) & L & -14 & -46 & -26 & 3.80 \\
\hline Cerebellar hemisphere (V) & L & -24 & -42 & -28 & 4.06 \\
\hline \multicolumn{6}{|l|}{ Cerebellar hemisphere } \\
\hline (VI) & L & -26 & -52 & -28 & 4.70 \\
\hline \multicolumn{6}{|l|}{ Cerebellar hemisphere } \\
\hline (VIII B) & $R$ & 18 & -60 & -48 & 6.25 \\
\hline & L & -20 & -62 & -52 & 4.90 \\
\hline
\end{tabular}

$t$ values and localizations (MNI coordinates) of activation peaks showing significantly $(p<0.05$; corrected for multiple comparisons) larger activation in the old group compared with the young group resulting from the following conjunction analysis: [(ISODIR-Rest) $)_{\text {old }}$ vs (ISODIR-Rest) $\left.{ }_{\text {young }}\right] \cap\left[\right.$ [NONISODIR-Rest) old $_{\text {VS }}$ (NONISODIRRest) young $_{\text {. }}$ L, Left hemisphere; $R$, right hemisphere; $M$, medial.

ditions in the elderly (blue) and younger (yellow) subjects. From these bar plots, it can be inferred that the BOLD responses in nearly all of the seven regions (Fig. $4 A-F$ ) were more or less equally high across the four movement conditions in the older group. In addition to these brain regions, the old group demonstrated significantly higher activation than the young group, which was specific to the coordination demands arising from combining hand and foot movements. These activations were located in the prefrontal cortex (PFC) (i.e., in the left inferior frontal sulcus), the ventral premotor cortex (PMv) (i.e., in the left inferior precentral sulcus) (Fig. $4 J$ ), the left and right superior frontal gyrus anterior from the ventral plane through the anterior commissure corresponding to the pre-SMA (Fig. $4 H$ ), the rostral part of the PMd $\sim 22 \mathrm{~mm}$ anterior to the movement-related activation in M1 corresponding to the pre-PMd (Fig. 4G), the right RCC (Fig. 4I), the left angular gyrus, the left anterior cerebellum (Fig. $4 K$ ), and the left and right posterior cerebellum (Fig. $4 L$, Table 3). The bar plots from Figure $4 G-L$ demonstrate that the BOLD responses in almost all of these regions increased for the coordination tasks and particularly when the more demanding NONISO condition was performed. This trend was observed for both groups but was much more pronounced in the older group.

No region was significantly more activated in the younger group than in the older group.

\section{Old-young group differences for ISODIR versus NONISODIR coordination}

Finally, we studied the between-group differences with respect to the comparison of nonisodirectional versus isodirectional hand- foot movements. The direct comparison of both coordination modes between older and younger subjects revealed an activation pattern in prefrontal, precentral, parietal, and occipital cortical regions as well as in the cerebellum (Fig. 5, Table 4). Some of these areas are typically activated during complex coordination tasks (i.e., left and right PMd) (Fig. 5A-C), left posterior parietal cortex, near the parieto-occipital fissure (Fig. 5C), and the cerebellar hemispheres and vermis (Fig. 5G,H) (Debaere et al., 2004; Wenderoth et al., 2004b). Furthermore, activation was observed in prefrontal areas [i.e., in the left anterior rostral cingulate cortex (aRCC)] (Fig. 5D), left superior frontal gyrus, corresponding to the pre-SMA (Fig. 5A), and middle frontal gyrus, corresponding to the dorsolateral prefrontal cortex (DLPFC) (Fig. 5D). Additional activation was also observed in the left insula (Fig. $5 F$ ). Finally, additional activation in the elderly was observed in the left and right middle occipital gyrus (Fig. 5E).

No region was significantly more activated in the younger group than in the older group.

\section{Discussion}

In the present study, we investigated age-related changes in the neural basis of cyclical isolated and coordinated hand-foot movements. Across all motor tasks, we found that the elderly exhibited stronger and more extended brain activation than the young subjects. The kinematic data suggested that task difficulty was reasonably matched between groups, such that the observed surplus activation in the elderly cannot only be explained by differences in motor output between both age groups. This additional recruitment is hypothesized to reflect compensation for age-related declines in neural functioning and/or difficulties in recruiting specialized neural mechanisms (Cabeza, 2002; Logan et al., 2002; Reuter-Lorenz, 2002; Buckner, 2004).

Below, we will focus on the direct comparison between young and old subjects and discuss the following three findings: (1) differential activations observed during both isolated and coordinated hand-foot movements, (2) differential activations that appear unique to coordinated hand-foot movements, and (3) differential activations during the direct comparison of the complex (NONISODIR) versus the simple (ISODIR) coordination mode.

\section{Additional brain activation in the elderly during both isolated and coordinated movements}

Regardless of the performed motor task, older subjects showed additional activation in large clusters surrounding the left frontal operculum, with peak activation in the anterior insular cortex, IFGPO, and superior temporal gyrus. This is in agreement with previous studies using auditory-paced movements, yielding higher frontal operculum activation in old compared with young subjects (Hutchinson et al., 2002). These areas seem to be involved in higher-order auditory processing (Platel et al., 1997; Bamiou et al., 2003; Thaut, 2003) and particularly in motor synchronization to an auditory rhythm (Thaut, 2003; Lewis et al., 2004). Accordingly, the observed additional activation around the frontal operculum suggests that the older subjects relied more pervasively on external information sources for controlling their limb movements by means of the metronome-pacing signal. This finding complies with the broader view that IFGPO is involved in interfacing external information about biological motion with internal representations of limb actions, as observed during movement observation, imitation, or imagery (Grezes et al., 1998; Iacoboni et al., 1999; Binkofski et al., 2000). Similarly, the fusiform gyri, which were activated in the elderly but not in the 


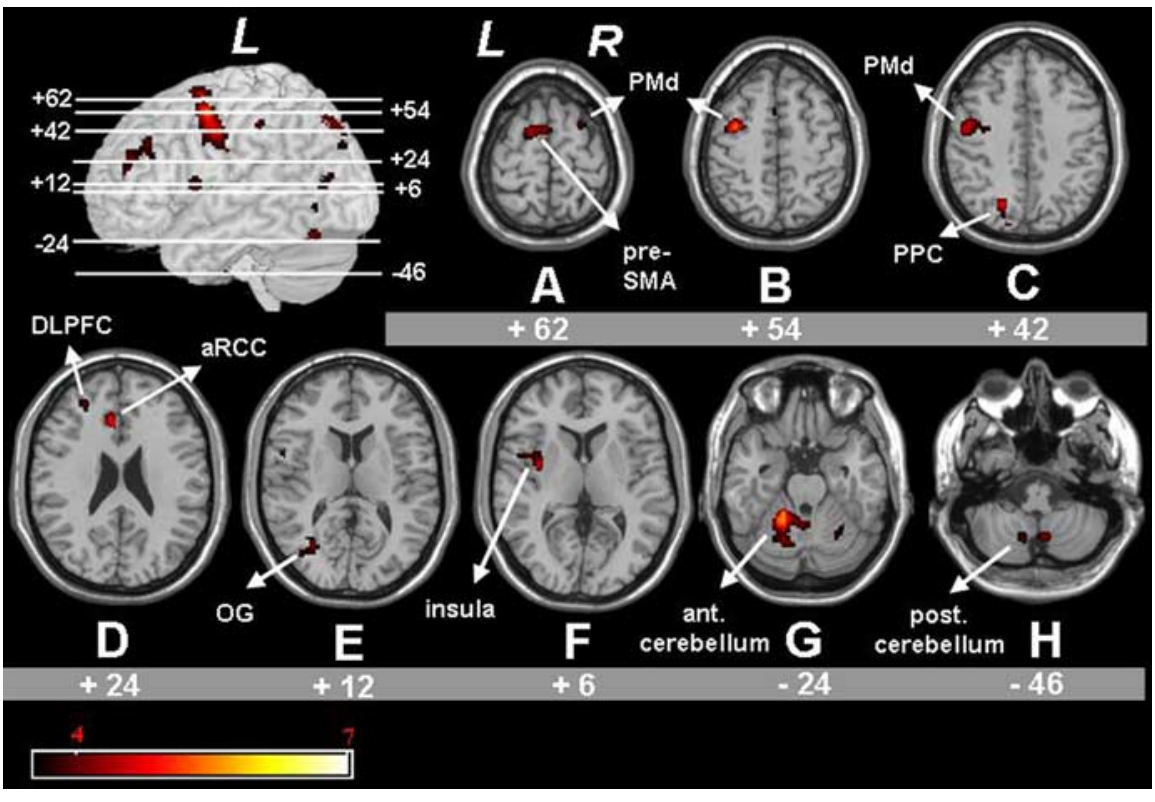

Figure 5. $\mathbf{A}-\boldsymbol{H}$, Statistical parametric mappings representing significantly larger activation in the old group compared with the young group with respect to the comparison of nonisodir versus isodir hand-foot coordination. significant voxels $(p<0.05$; corrected for multiple comparisons) are indicated in the red spectrum, and the height threshold is $t=3.60$. I, left hemisphere; $r$, right hemisphere; og, occipital gyrus; ppc, posterior parietal cortex; ant. cerebellum, anterior cerebellum; post. cerebellum, posterior cerebellum.

Table 4. Brain areas with significantly larger activation in the old group during the difficult NONISODIR versus the easy ISODIR coordination mode

\begin{tabular}{|c|c|c|c|c|c|}
\hline Area activated & Side & $x$ & $y$ & $z$ & $t$ value \\
\hline \multicolumn{6}{|l|}{ Middle frontal gyrus } \\
\hline (DLPFC) & $\mathrm{L}$ & -28 & 42 & 22 & 4.03 \\
\hline \multicolumn{6}{|l|}{ Anterior rostral cingulate } \\
\hline cortex & $\mathrm{L} / \mathrm{M}$ & -8 & 28 & 24 & 4.76 \\
\hline \multicolumn{6}{|l|}{ Superior frontal gyrus } \\
\hline (pre-SMA) & $\mathrm{L} / \mathrm{M}$ & -4 & 4 & 62 & 4.68 \\
\hline \multicolumn{6}{|l|}{ Superior frontal sulcus } \\
\hline \multirow[t]{2}{*}{ (PMd) } & $\mathrm{L}$ & -36 & -2 & 54 & 5.50 \\
\hline & $\mathrm{R}$ & 26 & 8 & 62 & 4.02 \\
\hline Precentral gyrus (PMd) & $L$ & -50 & -8 & 42 & 4.61 \\
\hline \multicolumn{6}{|l|}{ Fundus of central sulcus } \\
\hline (PMd) & $\mathrm{L}$ & -30 & -20 & 42 & 4.80 \\
\hline \multicolumn{6}{|l|}{ Inferior frontal gyrus pars } \\
\hline opercularis & $\mathrm{L}$ & -54 & 4 & 10 & 4.01 \\
\hline Insula & $\mathrm{L}$ & -36 & -2 & 6 & 4.66 \\
\hline Superior parietal gyrus & $L$ & -22 & -68 & 42 & 4.87 \\
\hline \multicolumn{6}{|l|}{ Precuneus (superior pari- } \\
\hline etal gyrus) & M & 2 & -50 & 62 & 4.30 \\
\hline \multirow[t]{3}{*}{ Middle occipital gyrus } & $\mathrm{R}$ & 24 & -78 & 2 & 4.27 \\
\hline & $\mathrm{L}$ & -38 & -66 & -2 & 3.91 \\
\hline & $\mathrm{L}$ & -32 & -68 & 12 & 4.18 \\
\hline \multirow[t]{2}{*}{ Cerebellar hemisphere (V) } & $\mathrm{L}$ & -22 & -50 & -20 & 7.00 \\
\hline & $\mathrm{R}$ & 24 & -60 & -26 & 3.86 \\
\hline Cerebellar vermis (VIII A) & $\mathrm{L} / \mathrm{M}$ & -8 & -66 & -36 & 4.95 \\
\hline Cerebellar vermis (IX) & $\mathrm{R} / \mathrm{M}$ & 6 & -62 & -46 & 4.32 \\
\hline
\end{tabular}

$t$ values and localizations (MNI coordinates) of activation peaks showing significantly $(p<0.05$; corrected for multiple comparisons) larger activation in the old group compared with the young group resulting from the following analysis: (NONISODIR-ISODIR) old versus (NONISODIR-ISODIR) young. L, Left hemisphere; R, right hemisphere; M, medial.

young subjects, have been discussed in relation to movement observation (Bonda et al., 1996; Grezes et al., 1998) and imagery (Jahn et al., 2004). Accordingly, the elevated activations in IFGPO and fusiform gyri are hypothesized to reflect the elderly's use of some form of visualization strategy to control their movements.

The elderly also exhibited additional activation in the left S2, left supramarginal gyrus, and right precuneus, consistent with previous results comparing the activation of old versus young subjects during various simple motor tasks (Hutchinson et al., 2002; Mattay et al., 2002; Ward and Frackowiak, 2003). These areas are known to be involved in the integration of somatosensory information to guide motor actions (Cavada and Goldman-Rakic, 1989; Kalaska et al., 1990; Ashe and Georgopoulos, 1994; Ferraina and Bianchi, 1994; Huttunen et al., 1996; Scott et al., 1997; Rizzolatti et al., 1998). Additionally, they seem to be related to the attentional effort required by a specific motor skill (Hari et al., 1990; Garcia-Larrea et al., 1991; Mima et al., 1998; Lam et al., 1999). These findings point to an increased focus on somatosensory processing to perform these rhythmical movements in the elderly.

Based on our findings, it appears that, even for the relatively simple movements, the elderly relied less than young subjects on automatized, internal movement generation. Instead, they activated additional areas reflecting higher-level processing and integration of auditory and somatosensory information to guide motor actions.

Coordination-specific additional activation in the elderly During coordinated hand-foot movements, elderly subjects, compared with young subjects, exhibited additional activation contralateral in the anterior and bilateral in the posterior cerebellum. The increased activation of the cerebellum is not surprising, because it is known to be involved in multilimb coordination (Debaere et al., 2001a, 2003, 2004; Ramnani et al., 2001; Tracy et al., 2001; Meyer-Lindenberg et al., 2002; Ullen et al., 2003), and it becomes increasingly activated when coordination complexity rises (Ullen et al., 2003; Debaere et al., 2004). It has been hypothesized that cerebellar activity reflects increasing demands on motor timing (Ivry, 1997; Mima et al., 1999; Habas et al., 2004; Wenderoth et al., 2004b) and/or sensory processing (Jueptner et al., 1997; Bushara et al., 2001; Thickbroom et al., 2003). Other coordination-specific activation in elderly subjects was observed in small spots in the PMv and in the left angular gyrus. Particularly, the PMv is activated during interlimb coordination (Ullen et al., 2003; Debaere et al., 2004).

Interestingly, other regions not considered to represent classical "coordination areas" also exhibited higher coordinationspecific activation in the elderly than in the young subjects. The following regions were all located in the frontal lobe: pre-SMA, pre-PMd, RCC, and PFC. Pre-SMA and pre-PMd activation have emerged previously in age-related comparisons of hand grip and visually paced button-press tasks (Mattay et al., 2002; Ward and Frackowiak, 2003). There is increasing agreement that these areas are more closely related to cognitive than to motor processes. More specifically, they are highly interconnected with the PFC (Keizer and Kuypers, 1989; Lu et al., 1994; Geyer et al., 2000) and become activated with increasing cognitive demands of a motor 
task (for review, see Picard and Strick, 2001). Interestingly, we have not observed activation of these areas in our previous coordination research on hand and/or foot movements in adolescents (Debaere et al., 2001a, 2003, 2004; Wenderoth et al., 2004a).

Overall, these findings suggest that, in the elderly, hand-food coordination strongly activated classical coordination areas but also several frontal regions interfacing motor control and cognition. This suggests that older subjects relied on increased cognitive monitoring of the more complex motor tasks, which is consistent with recent behavioral results (Holtzer et al., 2005).

\section{Additional brain activation in the elderly during complex versus simple coordination}

Old-young group differences were studied in a direct comparison of the NONISODIR versus the ISODIR coordination mode. On the basis of previous studies, we expected the more difficult NONISODIR mode to result in the recruitment of additional "coordination areas," such as SMA, PMd, the posterior parietal cortex, and the cerebellum (Sadato et al., 1997; Stephan et al., 1999; Debaere et al., 2001a; Immisch et al., 2001; Ehrsson et al., 2002). Indeed, all of these regions, except the SMA, were more activated in the elderly than in the young subjects during NONISODIR compared with ISODIR coordination.

However, in addition to these motor-specific areas, a nonmotor network also became activated in the elderly subjects. More specifically, we found increased activation in the DLPFC, preSMA, aRCC, left insula, and left and right middle occipital gyrus. Remarkably, all of the aforementioned regions were previously reported to be involved during conflict monitoring and/or inhibitory control, as assessed by Stroop, Go/NoGo, and Flanker tasks (Botvinick et al., 1999; Carter et al., 1999; Garavan et al., 1999; Casey et al., 2000; Kiehl et al., 2000; Menon et al., 2001; Watanabe et al., 2002). Particularly, the aRCC is generally active in error detection and in task conditions in which conflicting response tendencies need to be suppressed. Response inhibition has been shown to evoke a higher aRCC activity in old subjects compared with young subjects in cognitive tasks (Milham et al., 2002; Nielson et al., 2002). The additional aRCC activation in the elderly observed in our task is hypothesized to reflect a larger effort to suppress the regression to the ISODIR mode, which often emerges spontaneously during the less stable NONISODIR pattern (Baldissera et al., 1982). This represents an interesting example of generic brain areas that span the motor and cognitive domains.

\section{Conclusions}

The present study represents the first attempt to investigate the neural basis of age-related differences during rhythmical single limb and interlimb coordination patterns. All movements resulted in higher and more extended activation in the elderly compared with young subjects, although motor difficulty was matched between groups. Remarkably, no regions were significantly more activated in the younger group than in the older group. This differs from most studies on cognitive aging but is consistent with current research on motor performance (Calautti et al., 2001; Mattay et al., 2002; Ward and Frackowiak, 2003). The pattern of more extended activation in the elderly became more pronounced with increasing task complexity. For all motor tasks, the elderly exhibited additional activation in higher-level sensorimotor areas reflecting increased reliance on sensory information processing. During coordination, additional activation was also observed in areas reflecting increased cognitive monitoring of motor performance. In the most complex coordination task, the elderly exhibited activation in areas mainly involved in inhibitory cognitive control, as required for suppressing prepotent response tendencies.

Overall, our findings suggest that aging is associated with a shift along the continuum from automatic to more controlled information processing for movement, as reflected by more pronounced intersensory processing and integration of information, and increased attentional effort. The elderly's increased deployment of neural resources during motor performance, and particularly the recruitment of generic areas that span the motor and cognitive domains, may account for the extra difficulties they encounter during multitasking, such as thinking or talking while walking (Lindenberger et al., 2000; Li et al., 2001). As such, the present findings have theoretical as well as clinical relevance for gerontology.

\section{References}

Ashe J, Georgopoulos AP (1994) Movement parameters and neural activity in motor cortex and area 5. Cereb Cortex 4:590-600.

Baldissera F, Cavallari P, Civaschi P (1982) Preferential coupling between voluntary movements of ipsilateral limbs. Neurosci Lett 34:95-100.

Baldissera F, Cavallari P, Marini G, Tassone G (1991) Differential control of in-phase and anti-phase coupling of rhythmic movements of ipsilateral hand and foot. Exp Brain Res 83:375-380.

Bamiou DE, Musiek FE, Luxon LM (2003) The insula (Island of Reil) and its role in auditory processing. Literature review. Brain Res Brain Res Rev 42:143-154.

Binkofski F, Amunts K, Stephan KM, Posse S, Schormann T, Freund HJ, Zilles K, Seitz RJ (2000) Broca's region subserves imagery of motion: a combined cytoarchitectonic and fMRI study. Hum Brain Mapp 11:273-285.

Bonda E, Petrides M, Ostry D, Evans A (1996) Specific involvement of human parietal systems and the amygdala in the perception of biological motion. J Neurosci 16:3737-3744.

Botvinick M, Nystrom LE, Fissell K, Carter CS, Cohen JD (1999) Conflict monitoring versus selection-for-action in anterior cingulate cortex. Nature 402:179-181.

Buckner RL (2004) Memory and executive function in aging and AD: multiple factors that cause decline and reserve factors that compensate. Neuron 44:195-208.

Bushara KO, Wheat JM, Khan A, Mock BJ, Turski PA, Sorenson J, Brooks BR (2001) Multiple tactile maps in the human cerebellum. NeuroReport 12:2483-2486.

Cabeza R (2001) Cognitive neuroscience of aging: contributions of functional neuroimaging. Scand J Psychol 42:277-286.

Cabeza R (2002) Hemispheric asymmetry reduction in older adults: the HAROLD model. Psychol Aging 17:85-100.

Cabeza R, McIntosh AR, Tulving E, Nyberg L, Grady CL (1997) Age-related differences in effective neural connectivity during encoding and recall. NeuroReport 8:3479-3483.

Calautti C, Serrati C, Baron JC (2001) Effects of age on brain activation during auditory-cued thumb-to-index opposition: a positron emission tomography study. Stroke 32:139-146.

Carson RG, Goodman D, Kelso JA, Elliott D (1995) Phase transitions and critical fluctuations in rhythmic coordination of ipsilateral hand and foot. J Mot Behav 27:211-224.

Carter CS, Botvinick MM, Cohen JD (1999) The contribution of the anterior cingulate cortex to executive processes in cognition. Rev Neurosci 10:49-57.

Casey BJ, Thomas KM, Welsh TF, Badgaiyan RD, Eccard CH, Jennings JR, Crone EA (2000) Dissociation of response conflict, attentional selection, and expectancy with functional magnetic resonance imaging. Proc Natl Acad Sci USA 97:8728-8733.

Cavada C, Goldman-Rakic PS (1989) Posterior parietal cortex in rhesus monkey: II. Evidence for segregated corticocortical networks linking sensory and limbic areas with the frontal lobe. J Comp Neurol 287:422-445.

Debaere F, Swinnen SP, Beatse E, Sunaert S, Van Hecke P, Duysens J (2001a) Brain areas involved in interlimb coordination: a distributed network. NeuroImage 14:947-958.

Debaere F, Van Assche D, Kiekens C, Verschueren SM, Swinnen SP (2001b) 
Coordination of upper and lower limb segments: deficits on the ipsilesional side after unilateral stroke. Exp Brain Res 141:519-529.

Debaere F, Wenderoth N, Sunaert S, Van Hecke P, Swinnen SP (2003) Internal vs external generation of movements: differential neural pathways involved in bimanual coordination performed in the presence or absence of augmented visual feedback. NeuroImage 19:764-776.

Debaere F, Wenderoth N, Sunaert S, Van Hecke P, Swinnen SP (2004) Cerebellar and premotor function in bimanual coordination: parametric neural responses to spatiotemporal complexity and cycling frequency. NeuroImage 21:1416-1427.

de Rekeneire N, Visser M, Peila R, Nevitt MC, Cauley JA, Tylavsky FA, Simonsick EM, Harris TB (2003) Is a fall just a fall: correlates of falling in healthy older persons. The health, aging and body composition study. J Am Geriatr Soc 51:841-846.

Ehrsson HH, Kuhtz-Buschbeck JP, Forssberg H (2002) Brain regions controlling nonsynergistic versus synergistic movement of the digits: a functional magnetic resonance imaging study. J Neurosci 22:5074-5080.

Ferraina S, Bianchi L (1994) Posterior parietal cortex: functional properties of neurons in area 5 during an instructed-delay reaching task within different parts of space. Exp Brain Res 99:175-178.

Folstein MF, Folstein SE, McHugh PR (1975) "Mini-mental state." A practical method for grading the cognitive state of patients for the clinician. J Psychiatr Res 12:189-198.

Friston KJ, Holmes AP, Poline JB, Grasby PJ, Williams SC, Frackowiak RS, Turner R (1995a) Analysis of fMRI time-series revisited. NeuroImage 2:45-53.

Friston K, Holmes A, Worsley K, Poline J, Frith C, Frackowiak R (1995b) Statistical parametric maps in functional imaging: a general linear approach. Hum Brain Mapp 2:189-210.

Garavan H, Ross TJ, Stein EA (1999) Right hemispheric dominance of inhibitory control: an event-related functional MRI study. Proc Natl Acad Sci USA 96:8301-8306.

Garcia-Larrea L, Bastuji H, Mauguiere F (1991) Mapping study of somatosensory evoked potentials during selective spatial attention. Electroencephalogr Clin Neurophysiol 80:201-214.

Genovese CR, Lazar NA, Nichols T (2002) Thresholding of statistical maps in functional neuroimaging using the false discovery rate. NeuroImage 15:870-878.

Geyer S, Matelli M, Luppino G, Zilles K (2000) Functional neuroanatomy of the primate isocortical motor system. Anat Embryol (Berl) 202:443-474.

Grady CL (2000) Functional brain imaging and age-related changes in cognition. Biol Psychol 54:259-281.

Grady CL, Maisog JM, Horwitz B, Ungerleider LG, Mentis MJ, Salerno JA, Pietrini P, Wagner E, Haxby JV (1994) Age-related changes in cortical blood flow activation during visual processing of faces and location. J Neurosci 14:1450-1462.

Grezes J, Costes N, Decety J (1998) Top-down effect of strategy on the perception of human biological motion: a PET investigation. Cogn Neuropsychol 15:553-582.

Habas C, Axelrad H, Nguyen TH, Cabanis EA (2004) Specific neocerebellar activation during out-of-phase bimanual movements. NeuroReport 15:595-599.

Hari R, Hamalainen H, Hamalainen M, Kekoni J, Sams M, Tiihonen J (1990) Separate finger representations at the human second somatosensory cortex. Neuroscience 37:245-249.

Hedden T, Gabrieli JD (2004) Insights into the ageing mind: a view from cognitive neuroscience. Nat Rev Neurosci 5:87-96.

Heuninckx S, Debaere F, Wenderoth N, Verschueren S, Swinnen SP (2004) Ipsilateral coordination deficits and central processing requirements associated with coordination as a function of aging. J Gerontol B Psychol Sci Soc Sci 59:225-232.

Holtzer R, Stern Y, Rakitin BC (2005) Predicting age-related dual-task effects with individual differences on neuropsychological tests. Neuropsychology 19:18-27.

Hutchinson S, Kobayashi M, Horkan CM, Pascual-Leone A, Alexander MP, Schlaug G (2002) Age-related differences in movement representation. NeuroImage 17:1720-1728.

Huttunen J, Wikstrom H, Korvenoja A, Seppalainen AM, Aronen H, Ilmoniemi RJ (1996) Significance of the second somatosensory cortex in sensorimotor integration: enhancement of sensory responses during finger movements. NeuroReport 7:1009-1012.

Iacoboni M, Woods RP, Brass M, Bekkering H, Mazziotta JC, Rizzolatti G
(1999) Cortical mechanisms of human imitation. Science 286:2526-2528.

Immisch I, Waldvogel D, van Gelderen P, Hallett M (2001) The role of the medial wall and its anatomical variations for bimanual antiphase and in-phase movements. NeuroImage 14:674-684.

Ivry R (1997) Cerebellar timing systems. Int Rev Neurobiol 41:555-573.

Jahn K, Deutschlander A, Stephan T, Strupp M, Wiesmann M, Brandt T (2004) Brain activation patterns during imagined stance and locomotion in functional magnetic resonance imaging. NeuroImage 22:1722-1731.

Jueptner M, Ottinger S, Fellows SJ, Adamschewski J, Flerich L, Muller SP, Diener HC, Thilmann AF, Weiller C (1997) The relevance of sensory input for the cerebellar control of movements. NeuroImage 5:41-48.

Kalaska JF, Cohen DA, Prud'homme M, Hyde ML (1990) Parietal area 5 neuronal activity encodes movement kinematics, not movement dynamics. Exp Brain Res 80:351-364.

Keizer K, Kuypers HG (1989) Distribution of corticospinal neurons with collaterals to the lower brain stem reticular formation in monkey (Macaca fascicularis). Exp Brain Res 74:311-318.

Kelso JA, Jeka JJ (1992) Symmetry breaking dynamics of human multilimb coordination. J Exp Psychol Hum Percept Perform 18:645-668.

Kiehl KA, Liddle PF, Hopfinger JB (2000) Error processing and the rostral anterior cingulate: an event-related fMRI study. Psychophysiology $37: 216-223$.

Lam K, Kakigi R, Kaneoke Y, Naka D, Maeda K, Suzuki H (1999) Effects of visual and auditory stimulation on somatosensory evoked magnetic fields. Clin Neurophysiol 110:295-304.

Lewis PA, Wing AM, Pope PA, Praamstra P, Miall RC (2004) Brain activity correlates differentially with increasing temporal complexity of rhythms during initialisation, synchronisation, and continuation phases of paced finger tapping. Neuropsychologia 42:1301-1312.

Li KZ, Lindenberger U, Freund AM, Baltes PB (2001) Walking while memorizing: age-related differences in compensatory behavior. Psychol Sci 12:230-237.

Lindenberger U, Marsiske M, Baltes PB (2000) Memorizing while walking: increase in dual-task costs from young adulthood to old age. Psychol Aging 15:417-436.

Logan JM, Sanders AL, Snyder AZ, Morris JC, Buckner RL (2002) Underrecruitment and nonselective recruitment: dissociable neural mechanisms associated with aging. Neuron 33:827-840.

Lu MT, Preston JB, Strick PL (1994) Interconnections between the prefrontal cortex and the premotor areas in the frontal lobe. J Comp Neurol 341:375-392.

Mattay VS, Fera F, Tessitore A, Hariri AR, Das S, Callicott JH, Weinberger DR (2002) Neurophysiological correlates of age-related changes in human motor function. Neurology 58:630-635.

Menon V, Adleman NE, White CD, Glover GH, Reiss AL (2001) Errorrelated brain activation during a Go/NoGo response inhibition task. Hum Brain Mapp 12:131-143.

Meyer-Lindenberg A, Ziemann U, Hajak G, Cohen L, Berman KF (2002) Transitions between dynamical states of differing stability in the human brain. Proc Natl Acad Sci USA 99:10948-10953.

Milham MP, Erickson KI, Banich MT, Kramer AF, Webb A, Wszalek T, Cohen NJ (2002) Attentional control in the aging brain: insights from an fMRI study of the stroop task. Brain Cogn 49:277-296.

Mima T, Nagamine T, Nakamura K, Shibasaki H (1998) Attention modulates both primary and second somatosensory cortical activities in humans: a magnetoencephalographic study. J Neurophysiol 80:2215-2221.

Mima T, Sadato N, Yazawa S, Hanakawa T, Fukuyama H, Yonekura Y, Shibasaki H (1999) Brain structures related to active and passive finger movements in man. Brain 122:1989-1997.

Nielson KA, Langenecker SA, Garavan H (2002) Differences in the functional neuroanatomy of inhibitory control across the adult life span. Psychol Aging 17:56-71.

Oldfield RC (1971) The assessment and analysis of handedness: the Edinburgh inventory. Neuropsychologia 9:97-113.

Park HL, O'Connell JE, Thomson RG (2003) A systematic review of cognitive decline in the general elderly population. Int J Geriatr Psychiatry 18:1121-1134.

Picard N, Strick PL (2001) Imaging the premotor areas. Curr Opin Neurobiol 11:663-672.

Platel H, Price C, Baron JC, Wise R, Lambert J, Frackowiak RS, Lechevalier B, 
Eustache F (1997) The structural components of music perception. A functional anatomical study. Brain 120:229-243.

Ramnani N, Toni I, Passingham RE, Haggard P (2001) The cerebellum and parietal cortex play a specific role in coordination: a PET study. NeuroImage 14:899-911.

Reuter-Lorenz P (2002) New visions of the aging mind and brain. Trends Cogn Sci 6:394-400.

Rizzolatti G, Luppino G, Matelli M (1998) The organization of the cortical motor system: new concepts. Electroencephalogr Clin Neurophysiol 106:283-296.

Sadato N, Yonekura Y, Waki A, Yamada H, Ishii Y (1997) Role of the supplementary motor area and the right premotor cortex in the coordination of bimanual finger movements. J Neurosci 17:9667-9674.

Scott SH, Sergio LE, Kalaska JF (1997) Reaching movements with similar hand paths but different arm orientations. II. Activity of individual cells in dorsal premotor cortex and parietal area 5. J Neurophysiol 78:2413-2426.

Seidler RD, Stelmach GE (1995) Reduction in sensorimotor control with age. Quest 47:386-394.

Serrien DJ, Swinnen SP, Stelmach GE (2000) Age-related deterioration of coordinated interlimb behavior. J Gerontol B Psychol Sci Soc Sci 55B:295-303.

Spirduso WW (1982) Physical fitness in relation to motor aging. In: The aging motor system (Mortimer JA, Pirozzolo FJ, Maletta GJ, eds), pp 120-151. New York: Prager.

Spirduso WW (1995) Issues of quantity and quality of life. In: Physical dimensions of aging (Spirduso WW, ed), pp 5-30. Champaign, IL: Human Kinetics.

Stephan KM, Binkofski F, Halsband U, Dohle C, Wunderlich G, Schnitzler A, Tass P, Posse S, Herzog H, Sturm V, Zilles K, Seitz RJ, Freund HJ (1999) The role of ventral medial wall motor areas in bimanual co-ordination. A combined lesion and activation study. Brain 122:351-368.

Swinnen S, Jardin K, Meulenbroek R, Dounskaia N, Hofkens-Van Den
Brandt M (1997) Egocentric and allocentric constraints in the expression of patterns of interlimb coordination. J Cogn Neurosci 9:348-377.

Swinnen SP (2002) Intermanual coordination: from behavioural principles to neural-network interactions. Nat Rev Neurosci 3:350-361.

Talairach J, Tournoux P (1988) Co-planar stereotaxic atlas of the human brain. New York: Thieme.

Thaut MH (2003) Neural basis of rhythmic timing networks in the human brain. Ann NY Acad Sci 999:364-373.

Thickbroom GW, Byrnes ML, Mastaglia FL (2003) Dual representation of the hand in the cerebellum: activation with voluntary and passive finger movement. NeuroImage 18:670-674.

Tracy JI, Faro SS, Mohammed FB, Pinus AB, Madi SM, Laskas JW (2001) Cerebellar mediation of the complexity of bimanual compared to unimanual movements. Neurology 57:1862-1869.

Ullen F, Forssberg H, Ehrsson HH (2003) Neural networks for the coordination of the hands in time. J Neurophysiol 89:1126-1135.

Ward NS, Frackowiak RS (2003) Age-related changes in the neural correlates of motor performance. Brain 126:873-888.

Watanabe J, Sugiura M, Sato K, Sato Y, Maeda Y, Matsue Y, Fukuda H, Kawashima R (2002) The human prefrontal and parietal association cortices are involved in NO-GO performances: an event-related fMRI study. NeuroImage 17:1207-1216.

Welford AT (1988) Reaction time, speed of performance, and age. Ann NY Acad Sci 515:1-17.

Wenderoth N, Debaere F, Sunaert S, Van Hecke P, Swinnen SP (2004a) Parieto-premotor areas mediate directional interference during bimanual movements. Cereb Cortex 14:1153-1163.

Wenderoth N, Debaere F, Swinnen SP (2004b) Neural networks involved in cyclical interlimb coordination as revealed by medical imaging techniques. In: Neuro-behavioral determinants of interlimb coordination: a multidisciplinary approach. (Swinnen SP, Duysens J, eds), pp 127-222. Boston: Kluwer Academic. 